\section{Mild encephalopathy with reversible lesions in the splenium of corpus callosum and bilateral cerebral deep white matter in identical twins}

\author{
Junko Tahara,1 Jun Shinozuka,1 \\ Hitoshi Awaguni,1 Shin-ichiro Tanaka,1 \\ Shigeru Makino, ${ }^{1}$ Rikken Maruyama, ${ }^{1}$ \\ Shinsaku Imashuku² \\ 1Division of Pediatrics, 2Division of \\ Laboratory Medicine, Uji-Tokushukai \\ Medical Center, Uji, Kyoto, Japan
}

\section{Abstract}

Identical twin brothers developed mild encephalopathy at the age of 7.0 and 9.7 years (Patient 1) and 10.7 years (Patient 2). Patient 1 had influenza A at the time of his second episode, but triggering agents were not evident at the first episode. The triggering agents in Patient 2 were unclear. The neurological features of both patients included transient facial numbness, left arm paresis, dysarthria, and gait disturbance. Diffusion-weighted images from magnetic resonance imaging showed high signal levels at the splenium of corpus callosum and in the bilateral cerebral deep white matter. These results are characteristic of mild encephalitis/encephalopathy with a reversible isolated splenium of corpus callosum lesion. All three episodes were treated with a methylprednisolone pulse. Acyclovir was also administered to Patient 2 and to Patient 1 during his first episode. Patient 1 received an anti-influenza agent and intravenous immunoglobulin during his second episode. Both patients recovered completely without sequelae. Genetic factors, which may predispose identical twins to develop encephalopathy, are discussed.

\section{Introduction}

Reversible lesions in the splenium of the corpus callosum (SCC), detected using magnetic resonance imaging (MRI) with diffusionweighted imaging (DWI), have been associated with mild encephalitis/encephalopathy. This well recognized condition is classified as mild encephalitis/encephalopathy with a reversible isolated SCC lesion (MERS).1,2 The lesions are caused by various infectious agents, including influenza virus, rotavirus, Escherichia coli, mumps, and adenovirus. ${ }^{3}$ In addition, symmetrical reversible lesions in the cerebral deep white matter that are associated with those at the SCC have also been described in patients with MERS or those with influenza-associated encephalitis/encephalopathy (IAEE). ${ }^{3-6}$ IAEE patients exhibited mild clinical signs and symptoms and made a complete recovery, an outcome similar to that experienced by patients with MERS alone. MERS was the major abnormality noted in $15.5 \%$ (15/97) Japanese IEAA patients where brain MRI was performed and analyzed. ${ }^{7}$

We report three episodes of mild encephalopathy, including one of IAEE, in identical twins. The encephalopathy occurred between the ages of 7.0 to 10.7 years, and showed characteristic brain MRI features in the SCC and cerebral deep white matter. We also discuss possible factors that may increase the predisposition of identical twins to develop MERS or IAEE.

\section{Case Report \#1}

Patient 1 was the previously healthy older brother of identical twins. Aged 7.0 years, he complained of feeling ill, and exhibited lassitude of his left arm and gait disturbance. He was conscious and showed no abnormal neurological reflexes. Laboratory data, including those from blood and cerebrospinal fluid (CSF), were unremarkable. However, brain MRI with DWI showed the presence of high signals in the bilateral cerebral deep white matter that were suggestive of encephalopathy. No lesions at the SCC were noted (Figure 1). The exact infectious triggering agent(s) was unknown, although his anti-mycoplasma pneumoniae test was positive. He was treated with a methylprednisolone (mPSL) pulse followed by prednisolone tapering and acyclovir. He was later found to have a negative anti-herpes simplex virus (HSV)-IgM test result. His symptoms improved within a couple of days and a marked reduction of the signals in the follow-up brain MRI was present one week later. He lacked evidence of any seizures, although his electroencephalogram (EEG) was abnormal with spike waves at the bilateral parietal regions.

Patient 1 contracted influenza A when 9.7 years old. He became febrile $\left(37.8^{\circ} \mathrm{C}\right)$, temporalily drowsy, and was unable to walk or to communicate. Laboratory data, including blood and CSF results, were unremarkable. Brain MRI with DWI showed the presence of high signal lesions in the SCC and bilateral cerebral deep white matter indicating IAEE (Figure 1). He was treated with a peramivir hydrate (an antiinfluenza agent), a pulse of mPSL and intravenous immunoglobulin. His clinical symptoms improved rapidly within a couple of days. Reduced and vague high signals were, howev-
Correspondence: Shinsaku Imashuku, Consultant, Division of Laboratory Medicine, UjiTokushukai Medical Center, Uji, Kyoto 611-0042, Japan.

Tel.: +81.0774.20.1111 - Fax: +81.0774 .20 .2336 .

E-mail: shinim95@mbox.kyoto-inet.or.jp

Key words: Encephalopathy; Splenial lesion; Corpus callosum; Identical twins.

Acknowledgements: the authors thank Dr. Takeo Kato, Department of Pediatrics, Graduate School of Medicine and Faculty of Medicine Kyoto University, for his advice in the care of the patients.

Contribution: the authors contributed equally.

Conflict of interest: the authors declare no potential conflict of interest.

Received for publication: 21 May 2016.

Revision received: 3 August 2016.

Accepted for publication: 3 August 2016 .

This work is licensed under a Creative Commons Attribution NonCommercial 4.0 License (CC BYNC 4.0).

(C) Copyright J. Tahara et al., 2016

Licensee PAGEPress, Italy

Pediatric Reports 2016; 8:6615

doi:10.4081/pr.2016.6615

er, still present in the deep white matter in the follow-up brain MRI performed 3 months later (data not shown). The patient had no seizures despite having an abnormal EEG showing spike waves at the right parietal-temporal regions. He recovered completely without sequelae.

\section{Case Report \#2}

Patient 2 was the previously healthy younger identical twin brother, who aged 10.7 years developed transient facial palsy and loss of strength of his left arm. Laboratory data, including blood and CSF results, were unremarkable. Brain MRI with DWI, however, showed high signal lesions present in the SCC and bilateral cerebral deep white matter, results that were suggestive of encephalopathy (Figure 2). Although an anti-mycoplasma pneumonia test was positive, the exact infectious triggering agent(s) were unknown. He was treated with mPSL pulse therapy followed by prednisolone tapering and acyclovir. The clinical symptoms disappeared within a couple of days and he completely recovered with normal EEG and no sequelae. Follow-up brain MRIs performed one month later showed com- 
plete resolution of SCC but the presence of small remaining lesions in the deep white matter (data not shown). Both patients had a positive anti-nuclear antigen test; Patient 1 had $80 \times$ (normal $<40 \times$ ) and Patient 2 had $160 \times$ (normal $<40 \times$ ), respectively, but showed no symptoms of collagen disease.

Although MRI findings of our patients did not fit those of acute necrotizing encephalopathy (ANE), in order to further clarify the genetic predisposition, we proposed genetic analysis for the gene encoding nuclear pore protein Ran Binding Protein 2 (RANBP2), which has been reported in cases of recurrent or familial ANE; 8,9 however, the family declined the genetic studies.

\section{Discussion}

We report three episodes of mild encephalopathy in identical twins. Both exhibited brain MRI features that were characteristic of MERS associated with white matter lesions. Although the patients were treated with mPSL pulse therapy and, additionally, with anti-HSV or anti-influenza virus therapy, their neurological signs were transient, their clinical courses were mild, and they recovered completely without sequelae. The second episode in Patient 1 was clearly IAEE, but the precise etiology of the other two episodes was unknown. Laboratory data ruled out the possibility of HSV encephalitis, Reye's syndrome, or hereditary metabolic inborn errors.

Intramyelinic edema and/or inflammatory infiltrates are believed to be the cause of the high signal intensities in the SCC or white matter lesions shown using brain MRI with DWI. ${ }^{1}$ It should be emphasized that the white matter lesions are, like the SCC lesions, reversible with mild symptoms and patients show a good outcome.3-5 The white matter lesions may, however, cause a transient motor deficit. ${ }^{3}$ Indeed, both of our twins also had transient left arm paresis. Although our patients were treated with mPSL pulse therapy and/or acyclovir, there is controversy concerning the best way to treat patients with MERS. There is currently no specific therapy. There may be a causative cytokine effect on the development of MERS and some physicians prefer to employ steroid pulse therapy. ${ }^{10}$ This was the approach used in the current study. The outcome of MERS is generally excellent, but there is a report that MERS is not always reversible. ${ }^{11}$ Thus, the findings of the presence of small remaining lesions in the white matter at 1 to 3 months in the follow-up brain MRIs in our patients indicate the necessity of longterm careful observations.

The genetic factors involved in the development of MERS in identical twins remain unknown. In the past, MERS has been reported in sisters aged 2 and 6 years. ${ }^{12}$ Identical genetic susceptibility was suspected to have played a

role in cases of acute cerebellitis reported in 15-year-old twins. ${ }^{13}$ In terms of acute necrotizing encephalopathy (ANE), most cases are spo-

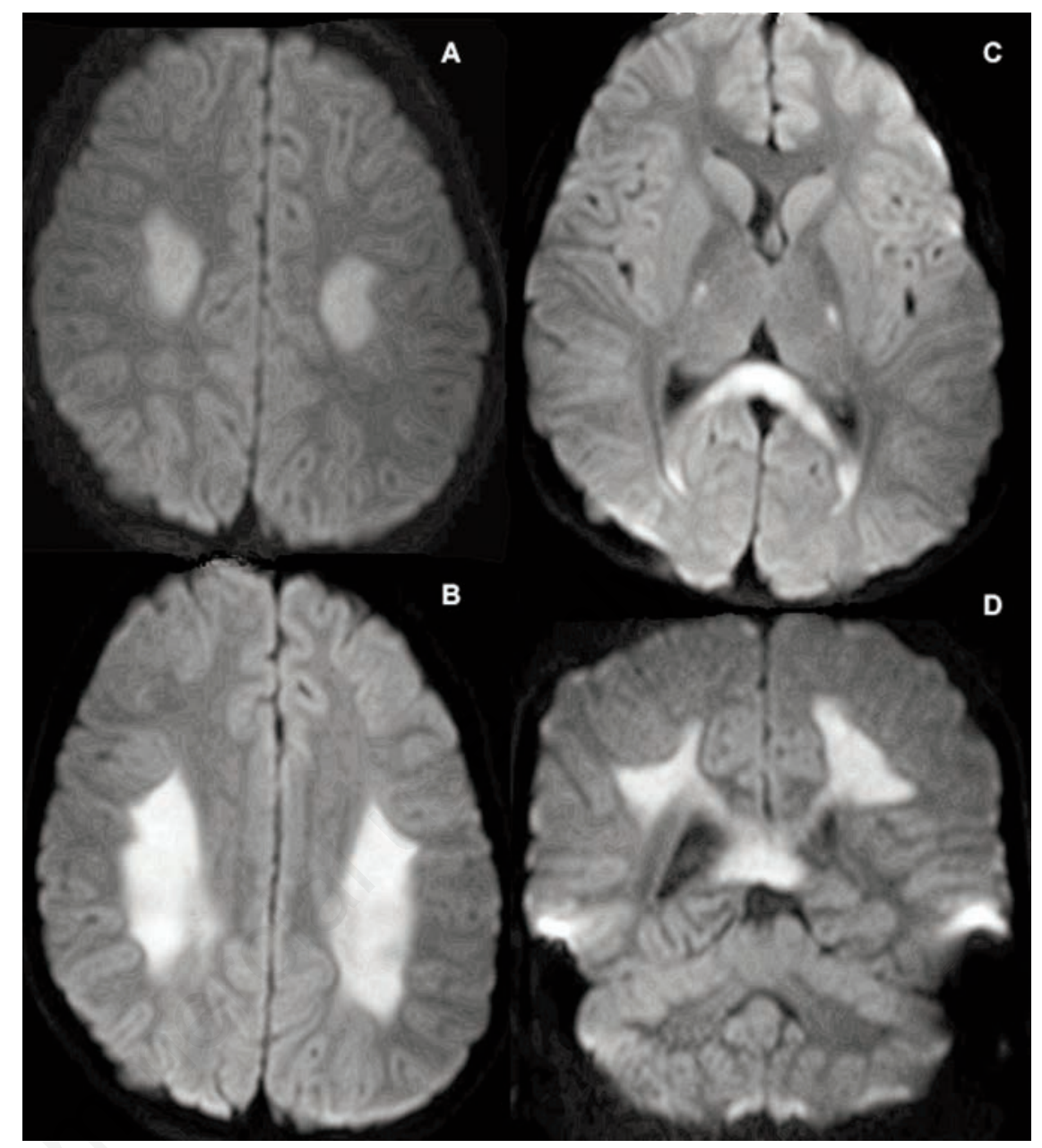

Figure 1. Brain magnetic resonance imaging (MRI) with diffusion-weighted images (DWI) shows the presence of high signals indicating lesions in the bilateral cerebral deep white matter (axial) alone in the first episode (A) and in the cerebral deep white matter (B; axial), at the splenium of the corpus callosum (SCC) (C; axial), and both (D; coronal) in the second episode (D) of the elder twin (Patient 1). Follow-up brain MRIs performed 3 months later showed complete resolution of lesions in the SCC but the presence of small remaining lesions in the deep white matter (data not shown).

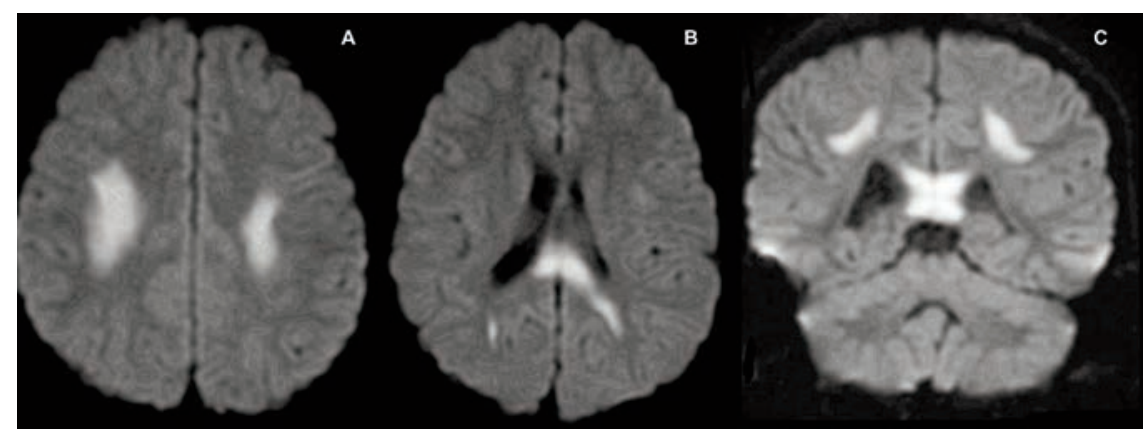

Figure 2. Brain magnetic resonance imaging (MRI) with diffusion-weighted images (DWI) shows the presence of high signals in the bilateral cerebral deep white matter (A; axial), at the splenium of the corpus callosum (SCC) (B; axial), and both (C; coronal) of the younger twin (Patient 2). Follow-up brain MRIs performed one month later showed complete resolution of SCC but the presence of small remaining lesions in the deep white matter (data not shown). 
radic and non-recurrent; however, familial cases or recurrent cases were rarely reported. ${ }^{8,9}$ Although MRI findings in our twins did not match with those in ANE with typical necrotizing lesions in the thalamus and brainstem, we wanted to clarify the pathogenesis in our twins with genetic analysis of RANBP2 to determine if familial MERS also show RANBP2 mutations. 8,9 Unfortunately, the family declined any genetic studies. There are reports of genetic factors linked with the occurrence of the same disease, although not neurological, in twins. Specific HLA typing was associated with the development of subacute thyroiditis or with focal myositis.14,15 However, no data are available showing the correlation between certain HLA typing and the occurrence of MERS to date.

\section{Conclusions}

In summary, we report MERS occurring in identical twins. Although common genetic backgrounds are suspected to be a factor in the predisposition to MERS, future studies are required to clarify the precise genetic factors in pathogenesis of MERS.

\section{References}

1. Takanashi J, Barkovich AJ, Yamaguchi K, Kohno Y. Influenza-associated encephali- tis/encephalopathy with a reversible lesion in the splenium of the corpus callosum: a case report and literature review. AJNR Am J Neuroradiol 2004;25:798-802.

2. Matsubara K, Kodera M, Nigami H, et al. Reversible splenial lesion in influenza virus encephalopathy. Pediatr Neurol 2007;37:431-4.

3. Bulakbasi N, Kocaoglu M, Tayfun C, Ucoz T. Transient splenial lesion of the corpus callosum in clinically mild influenza-associated encephalitis/encephalopathy. AJNR Am J Neuroradiol 2006;27:1983-6.

4. Takanashi J, Barkovich AJ, Shiihara T, et al. Widening spectrum of a reversible splenial lesion with transiently reduced diffusion. AJNR Am J Neuroradiol 2006;27:8368.

5. Cho JS, Ha SW, Han YS, et al. Mild encephalopathy with reversible lesion in the splenium of the corpus callosum and bilateral frontal white matter. J Clin Neurol 2007;3:53-6.

6. Ka A, Britton P, Troedson C, et al. Mild encephalopathy with reversible splenial lesion: an important differential of encephalitis. Eur J Paediatr Neurol 2015;19:377-82.

7. Ishida Y, Kawashima H, Morichi S, et al. Brain magnetic resonance imaging in acute phase of pandemic influenza $\mathrm{A}$ (H1N1) 2009: associated encephalopathy in children. Neuropediatrics 2015;46:20-5.

8. Neilson DE, Adams MD, Orr CM, et al. Infection-triggered familial or recurrent cases of acute necrotizing encephalopathy caused by mutations in a component of the nuclear pore, RANBP2. Am J Hum Genet 2009;84:44-51.

9. Denier C, Balu L, Husson B, et al. Familial acute necrotizing encephalopathy due to mutation in the RANBP2 gene. J Neurol Sci 2014;345:236-8.

10. Kimura E, Okamoto S, Uchida Y, et al. A reversible lesion of the corpus callosum splenium with adult influenza-associated encephalitis/encephalopathy: a case report. J Med Case Rep 2008;2:220.

11. Hashimoto Y, Takanashi J, Kaiho K, et al. A splenial lesion with transiently reduced diffusion in clinically mild encephalitis is not always reversible: a case report. Brain Dev 2009;31:710-2.

12. Imamura T, Takanashi J, Yasugi J, et al. Sisters with clinically mild encephalopathy with a reversible splenial lesion (MERS)like features; Familial MERS? J Neurol Sci 2010;290:153-6.

13. Xu F, Ren SQ, Liu JY. Acute cerebellitis in identical twins. Pediatr Neurol 2008;39:432-4.

14. Hamaguchi E, Nishimura Y, Kaneko S, Takamura T. Subacute thyroiditis developed in identical twins two years apart. Endocr J 2005;52:559-62.

15. Sekiguchi K, Kanda F, Oishi K, et al. HLA typing in focal myositis. J Neurol Sci 2004;227:21-5. 